\title{
METHODS OF ANALYSING THE VIRULENCE OF BACTERIA AND VIRUSES FOR GENETICAL PURPOSES
}

\author{
By LUIGI L. CAVALLI and GIOVANNI MAGNI \\ Sezione Genetico Istituto Itoliono di Idrobiologio, Pollonzo (Novara) and \\ Istituto di Anatomio Patologico Universito, Milono
}

Received 13.xii.46

RECENT investigations, published in English during the war years, have made considerable progress in the genetical analysis of the host-parasite system (literature given by Gowen and Zelle); but so far as we have been able to ascertain from the literature available to us, the measure of virulence used in this work has been based largely on the percentage survival of host individuals after experimental infection.

In Continental Europe the genetical aspects of host-parasite interaction have not yet been studied, but progress has been made in regard to the measurement of virulence which, we believe, will prove useful in genetical studies. We refer to the measure of virulence based upon observations of the individual death times of inoculated animals instead of on the percentage survival.

Theoretically the measurement of death times has advantages over the percentage of death or survival. First of all, it leads to two statistics from any set of experimental data; the mean death time, which, if there is partial survival (the mean then being estimated as the median), is correlated with the percentage of deaths; and the variance of death times, which is an estimate of the variability of the population unobtainable from percentage data alone. This variance and the form of the distribution of death times have both a biological and a genetical meaning. The measurement of individual death times permits the recovery of both extra statistical and extra genetical information. Statistically the precision of the experimental results may be doubled in this way. Accounts of this method and the results of its application have appeared in Italian and German during the war. We hope that this note will serve to bring them to the notice of those English-speaking scientists who might otherwise overlook them.

The study of death times has revealed the existence of a linear relation between mean death time and the logarithm of the number of bacteria used for inoculations, over a wide range. We have shown this relation to hold in experiments on the infection of white mice with Pneumococcus type I and with $B$. anthracis (see fig. I). The data of other investigators have given the same result for the tuberculous 
infection of guinea-pigs (Prigge), and for at least the highly virulent strains of Salmonella typhimurium in mice (Pike and Mackenzie). A linear relation of mean death time and $\log$ dose has also been found with virus infection, as with the pneumonitis virus of mice (Goennert) and typhus fever virus in mice (Ipsen).

Death time, $t$, can thus be related to dose, $d$, by the equation

$$
t=-a \log d+b \text {. }
$$

Ipsen has proposed a more complicated equation which takes into account the asymptotic approach of death time to a value independent of dose, at the higher dosages (see, for example, the left curve of fig. I).

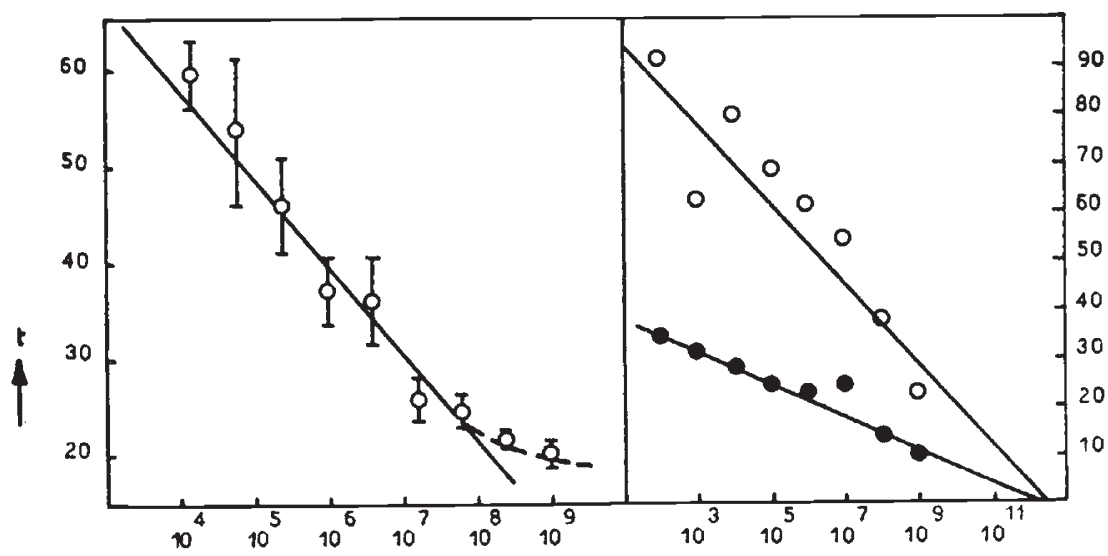

FIG. I.-Left. Arithmetic mean death times in hours (with standard errors) of white mice injected with $B$. anthracis, plotted against number of bacteria used in the inoculation on a logarithmic scale. Data from Bonezzi, Cavalli and Magni.

Right. Harmonic mean death times of white mice inoculated with pneumococcus type I plotted against dose on a logarithmic scale as before. Each point is the mean of 12 mice. Data from Cavalli and Magni.

Filled circles : untreated mice.

Open circles : mice treated with $0.6 \mathrm{mg} . / \mathrm{g}$. sulfapyridine.

The equation relating death time linearly to log dose may have a simple interpretation on the assumptions (i) that the multiplication of the infectant micro-organism is logarithmic within the host, and (ii) that death takes place when a certain number of micro-organisms are present in the body, irrespective of the initial dose itself. A simple elaboration of this hypothesis allows us to cover the case of the destruction of the infecting organisms, without any great change in the relation of death time to dose. According to the simpler interpretation the constant $a$, measuring the slope of the line, is 3.3 times the generation time (the reciprocal of the reproduction rate) of the infecting micro-organism, while $b$ is directly proportional to the $\log$ of the number of organisms which exist at the moment of death in the host. The constant $b$ is therefore correlated with the toxicity of the bacterial strain used. The actual $\log$ of the number 
of organisms giving death may be estimated as $b / a$, i.e. as the segment between the origin and the point at which the line cuts the abscissa.

While we should probably regard this interpretation as an oversimplified account of the processes occurring in the host, it is at least a first approximation to any more complete account, which must still lead to a simple relation between death time and dose. That our interpretation is at least a useful approximation is shown by the fact that it has been possible to confirm by experiment some of its implications, in particular the relation between the constant $a$ and the reproduction rate. Animals treated with sulfonamides and infected with different doses of pneumococci still give the linear relation of death time to dose, but with the constant $a$ changed in a way which, in our view, indicates a marked decrease of reproduction rate. We expect the ratio $b / a$ to depend on the toxicity of the micro-organism, and to be independent of the reproduction rate, and it was in fact unchanged or changed only slightly by the use of sulfonamides (see fig. I, curves on the right).

Ipsen affords a more direct test of the hypothesis by providing the means of evaluating the reproduction rate of typhus fever virus both from equation (I) and from the observed growth curve of the virus in the host. The two values so obtained agree within the limits of experimental error.

Further tests of the hypothesis have not yet been made, but we believe that they will reveal no serious difficulties. The importance of the hypothesis is that the scheme of analysis to which it leads, isolates two factors of virulence, the reproduction rate and the toxicity of the strain. Interactions with natural and acquired immunity mechanisms are yet to be examined.

The relation between death time and dose takes no account of the distribution of individual death times of animals inoculated with the same dose of micro-organisms. Such distributions seem to differ with different infections. The distributions of death times of tuberculosis in guinea-pigs, for example, seem to be nearly normal (data from Prigge), while those of white mice infected with pneumococci and streptococci are skewed, becoming normal if the reciprocals of death times (Prigge's " death velocities") are plotted in place of the death times themselves (see figs. 2, 3 and 4). Ipsen makes use of the device, for normalising the distribution (previously employed by him for titrating the bacterial toxins on the basis of death times), of introducing a function, $f(T)$, of the times which follows from his equations relating death time to dose. Distributions of death times, or of functions of them, obviously have a genetical significance, and will show the effects of gene segregations.

Partial survival introduces difficulties into the statistical treatment. The methods proposed for the handling of "truncated " distributions (as for example by Bliss) cannot always be applied directly because, as we shall see in discussing fig. 3 , they may result in a loss of biological 
information. This occurs when the distribution is not completely rectilinear up to the breakage point, corresponding to the percentage

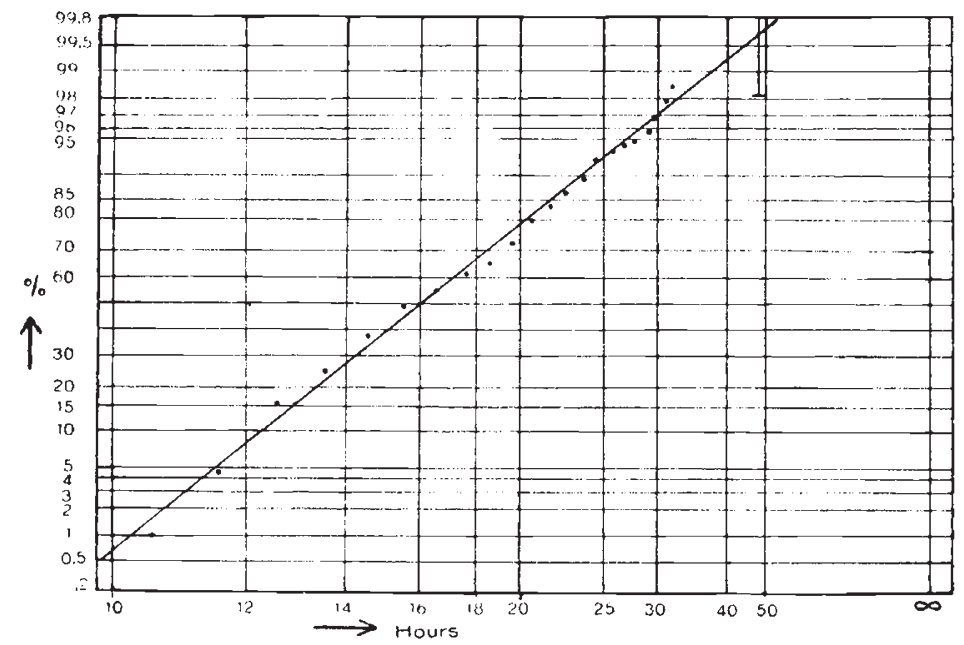

FIG. 2.-Distribution of death times, on a reciprocal scale, of 191 white mice each inoculated i.p. with $10^{8}$ pneumococci type I. Plotted on probability paper. Data from Cavalli and Magni.

of mortality when plotted on probability paper with a suitably transformed scale along the abscissa (see fig. 3). The use of reciprocal death times, with the calculation of harmonic means, allows the

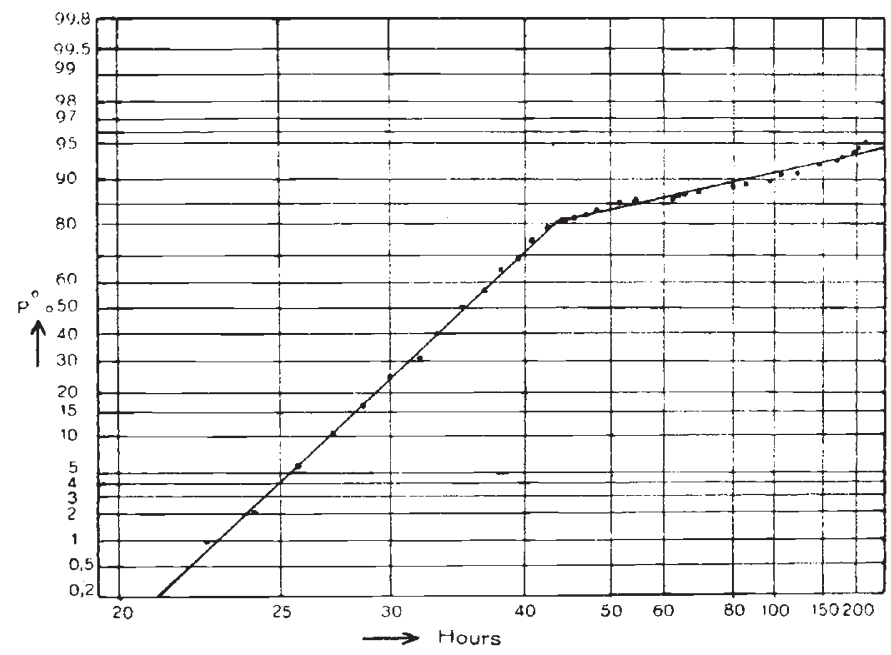

FIG. 3.-Distribution of death times, on a reciprocal scale, of I 92 white mice each inoculated i.p. with Io $^{2}$ pneumococci type 1. Plotted on probability paper. Data from Cavalli and Magni.

survivors to be taken into consideration, but it too fails to give fully satisfactory results. 
An interesting case of partial survival is shown in fig. 3. The dose of pneumococci was low and so permitted partial survival. When plotted on probability paper the slope of the distribution showed a sudden change at one point. This could not be due to heterogeneity of the test animals, as bimodal distributions do not have this form on probability paper. We have assumed, as a working hypothesis, that the change is due to the appearance of specific antibodies, permitting survival of the inoculated animals, or at least survival for a longer time than would have been possible in the absence of antibodies.

If this hypothesis should prove true, either the statistical analysis should be limited to the first part of the distribution, the consequences of the specific immunity being so eliminated, or it must be elaborated

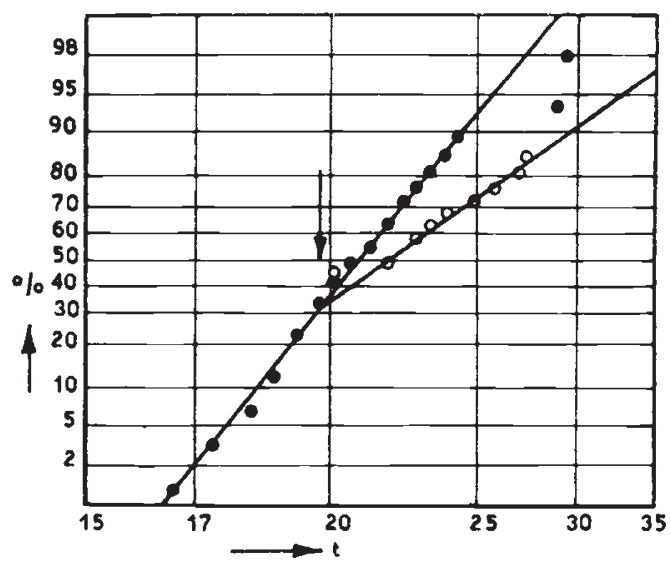

Fig. 4--Distribution of death times, on a reciprocal scale, of white mice inoculated with hæmolytic streptococci, plotted on probability paper. At the time marked by the arrow, half of the survivors were injected with sulfapyridine (open circles).

to cope with the effects of the immunity as expressed in the second part of the distribution.

The sensitivity of the method of plotting the distribution on probability paper, for the elucidation and analysis of variation in individual resistance, is well shown by an experiment testing the effect of injecting sulfonamide in a late phase of infection. Forty-six white mice were given a uniform dose of hæmolytic streptococci, low, but sufficient to bring about Ioo per cent. mortality. At $19 \frac{1}{2}$ hours after the experiment started, when 18 of the animals were dead, 14 of the remainder were injected with $0.6 \mathrm{mg}$./g. sulfapyridine each, the other I4 being left untreated. None of the untreated mice survived, but 3 survived out of the I4 given sulfonamide. The time mortality curves are plotted on probability paper in fig. 4. The I4 untreated animals continue the line of the first 18 to die; but the sulfonamide injection causes a break in the distribution. The 14 treated mice fall on a line which, though still approximately straight, 
is less steep than that of the other 32 . The slopes of the two lines are inversely proportional to their standard deviations. So the ratio of the slope from the 32 untreated mice to the slope from the treated I4, when squared, gives a variance ratio which, with $n_{1}=\mathrm{I} 3$ and $n_{2}=3 \mathrm{I}$ degrees of freedom, is significant at the I per cent. level. The potencies of chemotherapeutic drugs can be compared in this way.

This research and its application to genetical problems of the host-parasite systems are now held up by lack of financial means; but the results so far achieved by using death times have shown how at least two major factors of virulence, reproduction rate and toxicity, may be separated. Doubtless further virulence factors will be isolated and measured as the method of analysis is extended.

Finally, the use of death times, as opposed to death or survival percentages, permits us to employ infections of highly virulent microorganisms and so to eliminate, or at least minimise, disturbances arising from specific immunity or allergy. The initial interpretation is thereby simplified and made into a surer basis for the eventual consideration of the more complex cases. These complications may not be so great as might be feared, for infections such as tuberculosis, whose lethal effects are slow, appear to give the same relation between dose and time of death.

\section{REFERENCES}

Burss, G. I. . . . . . . . . 1937. Ann. appl. Biol., 24, 815-852.

Bonezzi, G., Cavalli, L. L., and 1943. Zbl. Bakt., I. Orig., 150, 17-25. MAGNI, G.

Gavalli, L. L., and Magni, G. . . 1942. Boll. d. Soc. Med. Chir. Pavia, 20, $609-624$.

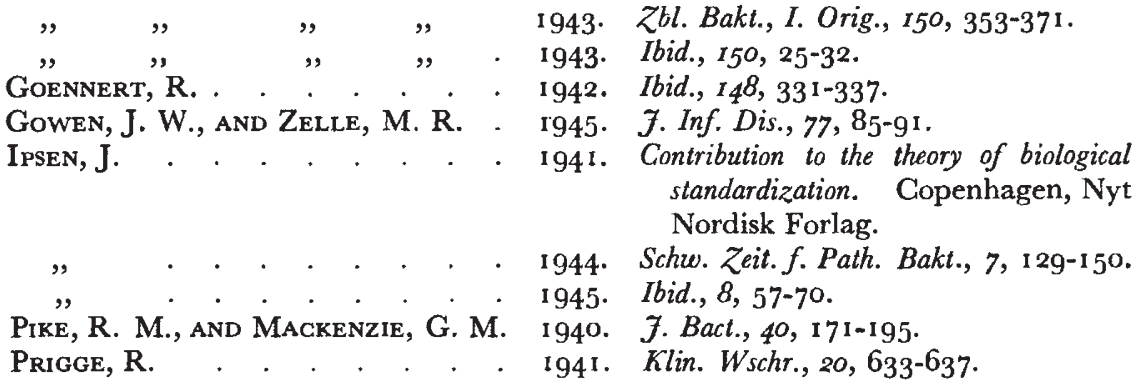

